\title{
Ein neues Web-Journal für Schulleiter*innen aus Österreich und der Schweiz stellt sich vor
}

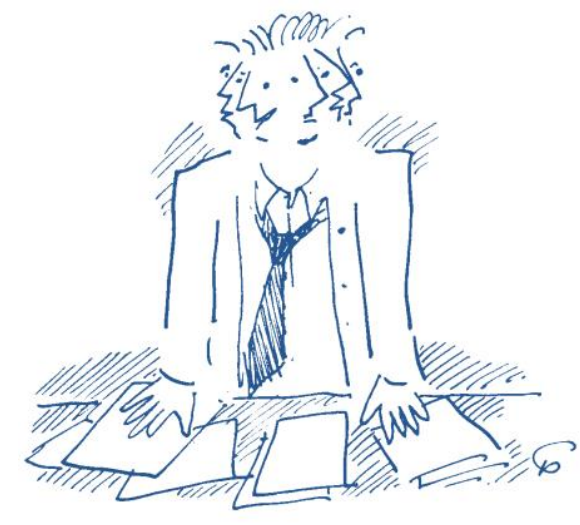

Dieses nachfolgend geführte "Gespräch" ist ein bloß fiktives, es will doch ein informatives sein. Es soll anregen zu weiteren Rückfragen, Rückmeldungen und Wunschäußerungen aller Art an dieses Web-Journal, seine Redaktion und seine Herausgeber*innen.

Alle Schulleiter*innen seien herzlich eingeladen, sich zu beteiligen - am besten mit eigenen Beiträgen in einer unserer Rubriken des Web-Journals! Gerne auch durch Beteiligung an unseren Online-Web-Dialogen, die wir im Kontext des Web-Journals in regelmäßigen Abständen anbieten. Kritik und Vorschläge zur weiteren Entwicklung unserer Vorhaben werden selbstverständlich auch gerne entgegengenommen.

Fragende*r ist ein*e imaginäre*r Schulleiter*in, es antwortet ein Herausgeber und Planender von \#schuleverantworten.

\begin{abstract}
Wir Schulleiter*innen stöhnen unter den extremen Corona-Bedingungen und den damit verbundenen Unsicherheiten. Und Ihnen an den Hochschulen fällt nichts Besseres ein als neues Lesefutter?
\end{abstract}

Erwin Rauscher: Haben Sie keine Sorge - stöhnen gehört doch zum guten Ton. Wer heute nicht stöhnt, der schläft. Und verschläft eine Entwicklung nicht nur der Schulpädagogik, die unumkehrbar ist und uns vermutlich noch lange nach der COVID-19-Pandemie beschäftigen wird: Digitalität. Und Sozialität als deren nur scheinbarer Antagonist. Dagegen halte ich ein Plakat des Roten Kreuzes, das für unsere Krisenzeit durch COVID-19 ausweist: Social Distancing muss heißen: Physical Distancing und Social Solidarity. Wir wollen einen zugegeben virtuellen - Ort der Solidarität, der Kultur, der Begegnung, des Gesprächs und des Optimismus schaffen. Und dafür haben wir als Medium dieses Web-Journal entwickelt und bieten es nunmehr allen Schulleiter*innen aus Österreich und der Schweiz an.

Wäre es nicht viel wichtiger, den Schulleiter*innen gerade in Krisenzeiten mehr Sicherheit anzubieten?

Das ist zweifellos eine wichtige und richtige Forderung, denn ohne Sicherheit gibt es kaum Freiheit. Aber lassen Sie mich die Gegenfrage stellen: Ist nicht jene Tür die sicherste, die man 
offen halten kann? Und haben gerade jene, die ihre Freiheit aufgeben, wirklich mehr Sicherheit? Anders gesagt: Wir möchten Schulleiter*innen (auch über die Landesgrenzen hinaus) miteinander ins Gespräch bringen, Ideen transportieren aus Good Practice und Next Practice, Entscheidungshilfen anbieten und Stellung beziehen zu aktuellen Herausforderungen im Kontext von Schule und Gesellschaft.

Für Leiter*innen welcher der unterschiedlichen Schulformen bieten Sie dieses Journal an?

Uns erfreut Vielfalt! Wir möchten unsere Beiträge zielgerichtet für jede Schulform anbieten, aber im offenen Dialog. Als man einen Spartaner gefragt hat, wie weit sein Horizont reiche, soll er geantwortet haben: ,So weit wie meine Speere.' Wir wollen den Horizont der je eigenen Schulform bewusst überschreiten, um miteinander ins Gespräch zu kommen und voneinander lernen zu können.

\section{Welche Beiträge wollen Sie dann in Ihrem Web-Journal präsentieren?}

Unsere Beiträge sind gegliedert in Rubriken, also nach einheitlich und deshalb wiedererkennbaren, feststehenden Gliederungen. Diese nennen wir ,Kategorien' - zugegeben ein wenig in Anlehnung an Immanuel Kant, für den diese als Denkformen zur Grundvoraussetzung von Erfahrung werden. Sie sind einerseits offen für (fast) alles, andererseits aber leicht zuzuordnen: Mit ,Aus der Praxis' berichten wir von schulischen Projekten, um aus Erfahrung lernen zu können. Bei ,Im Gespräch mit'geben wir Impulse, Ideen und Anregungen weiter, auch von Menschen mit anderen als pädagogischen Berufsfeldern. Mit ,Kultur macht Schule' berichten wir beispielhaft aus der Welt der Kultur für den Kosmos der Schulkultur. In ,Lesen lohnt sich' rezensieren wir Bücher und geben aktuelle Hinweise auf Lesenswertes. Jedes Heft enthält eine ,Begriffsbox', in der zum Schwerpunkt der jeweiligen Ausgabe eine präzise Erklärung angestrebt ist. ,pro \& contra' dient dazu, unterschiedliche Positionen wie in Leserbriefen zu Wort kommen zu lassen. Und, Führungskultur' schießlich wird Impulse enthalten, die uns als Schulleiter*innen immer wieder neu bewusst machen wollen, welche Verantwortung wir tragen. Ein an den Anfang gestelltes Editorial schließlich soll in das jeweilige Schwerpunktthema einführen.

Da haben Sie sich aber viel vorgenommen! Und warum dürfen diese Texte nur Schulleitungspersonen lesen und abonnieren?

Wir richten unser Angebot gezielt an Schulleiter*innen - hier sind wir der Meinung, dass es speziell für diese bisher zu wenige Angebote gibt. Aber selbstverständlich können alle, die Interesse haben, dieses Journal lesen und auch abonnieren - wir leben und geben open access!

Wenn man \#schuleverantworten abonniert, welche Kosten und welcher Nutzen entstehen?

Kosten? Keine! Unser Angebot ist kostenlos und zugangsfrei, aber nicht umsonst: Denn wir wünschen uns von allen unseren Leser*innen Mit- und Zusammenarbeit, aus den Schulen und für diese! Je mehr Beteiligung wir erzielen, die von Schule betroffen sind, umso mehr wird ihnen und uns allen dieses Journal von Nutzen sein können. Dafür planen wir vier 
reguläre Ausgaben jährlich und sind offen für zusätzliche Sondernummern bei Bedarf und aus aktuellen Gründen.

\section{Warum wird das Journal für Schweizer und für österreichische Schulleiter*innen angeboten?}

Das Web-Journal richtet sich auch an Schulleiter*innen anderer Länder, wird aber von der Pädagogischen Hochschule Niederösterreich und der Pädagogischen Hochschule Zürich gemeinsam angeboten und fokussiert deshalb vorrangig an Schulverantwortliche in diesen beiden Ländern.

\section{Noch eine letzte Frage: Was wünschen Sie sich als Herausgeber für dieses Web-Journal?}

Es soll keine Zeitschrift über die Schule entstehen, sondern eine für die Schule und für die darin Hauptverantwortlichen. Und für die wichtigste Ursache von Bildung, das Lernen. Denn Schule sind WIR, Schule geht uns alle an. Wir möchten ihr Wert zuschreiben, uns für sie mitverantwortlich fühlen. Ich glaube an die Schule als einen Ort der Bildung für jene Menschen, auf die wir hoffen!

Mit Erwin Rauscher sprach der*die (imaginäre) Schulleiter*in N.N. 